Sādhanā Vol. 36, Part 3, June 2011, pp. 339-348. (c) Indian Academy of Sciences

\title{
On the method of calibration of the energy dispersive EXAFS beamline at Indus-2 and fitting theoretical model to the EXAFS spectrum
}

\author{
ABHIJEET GAUR $^{\mathrm{a}, *}$, AJITA JOHARI $^{\mathrm{a}}$, B D SHRIVASTAVA ${ }^{\mathrm{a}}$, \\ D C GAUR $^{\mathrm{b}}, \mathrm{S}^{\mathrm{N}} \mathrm{JHA}^{\mathrm{c}}$, D BHATTACHARYYA ${ }^{\mathrm{c}}$, A POSWAL $^{\mathrm{c}}$ \\ and $S K \mathrm{KEB}^{\mathrm{d}}$
}

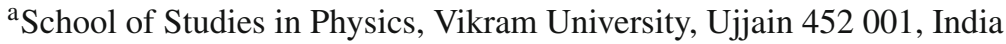

${ }^{\mathrm{b}}$ Government P G College Rajgarh, Biaora 465 611, India

${ }^{\mathrm{c}}$ Applied Spectroscopy Division, Bhabha Atomic Research Centre, Mumbai 400 085, India

${ }^{\mathrm{d}}$ Indus Synchrotron Utilization Division, Raja Ramanna Center for Advanced Technology, Indore 452 013, India

e-mail: abhijeetgaur9@gmail.com

MS received 27 August 2010; accepted 14 December 2010

\begin{abstract}
A procedure for calibration of the recently developed energy dispersive EXAFS beamline at the Indus- 2 synchrotron source at RRCAT, Indore, India has been described. The procedure involves recording of absorption spectra of two standard samples, whose absorption edge energies are well-established. Two methods have been considered for calibration. In the first method, the position of the first maximum of the derivative of absorption curve is taken as the position of the edge energy. In the second method, the position of the point at half edge step in the absorption curve is taken as the position of the edge energy. It has been shown that only the first method gives same values of dispersion even when the beam current is varied and should be used for calibrating the experimental spectra. Further, it is recommended that the performance of the beamline for a particular setting should be checked by recording and analysing EXAFS of a standard. Hence, the procedure for analysis and extracting information about the various parameters that can be determined by fitting the EXAFS data with a theoretical model has also been described, by taking the example of $\mathrm{K}$-absorption spectra of copper metal foil recorded on this beamline.
\end{abstract}

Keywords. Energy dispersive EXAFS; calibration method; theoretical model; $\mathrm{Cu}$ metal EXAFS.

*For correspondence 


\section{Introduction}

With the advent of the modern bright synchrotron radiation sources, extended X-ray absorption fine structure (EXAFS) spectroscopy has emerged out to be one of the powerful structure determination techniques that can be applied to any type of material, e.g., amorphous solids, liquids, solutions, gases, polymers and surfaces. EXAFS is an important probe for materials, since knowledge of local atomic structure, i.e., the species of atoms present and their locations, essential to progress in many scientific fields, whether for biology, chemistry, electronics, geophysics, metallurgy, or materials science. (Lytle 1999; Stern 2001; Tournus et al 2002; Hricovini et al 2003; Ascone et al 2003).

EXAFS measurements with synchrotron radiation are generally carried out on two different types of beamline set-ups. In the first type of EXAFS beamline set-up, a Double Crystal Monochromator (DCM) is used which selects a particular energy from the incident synchrotron beam. The monochromatic beam is made incident on the sample and the intensity of the transmitted beam passing through the sample or that of the fluorescence beam emerging out of the sample is recorded along with the incident intensity at each energy by scanning the DCM. EXAFS can be measured in transmission, fluorescence, and electron yield modes. Detectors that can be used are ionization chambers, Peltier-cooled $\mathrm{Si}(\mathrm{Li})$ detectors, multi-element germanium diode array fluorescence detectors, Lytle fluorescence and electron yield detectors.

In the second type of EXAFS beamline set-up, a bent crystal polychromator is used, to select a band of energy from the white synchrotron beam, which is horizontally dispersed and focused on the sample. The transmitted beam intensity from the sample is recorded on a position sensitive CCD detector, thus enabling recording of the whole EXAFS spectrum around an absorption edge in a single shot. The main advantages of dispersive EXAFS set-up are the focusing optics, short acquisition time (few ms) and great stability during the measurements due to the absence of any mechanical movement. Thus, in situ and time-resolved experiments can be easily performed on such kind of set-ups (Lamberti et al 2003, Smolentsev et al 2009).

This second type of EXAFS beamline set-up has been recently developed at the Indus-2 synchrotron source at RRCAT, Indore, India (Das et al 2004, Bhattacharyya et al 2009, Bhattacharyya et al 2009). On this beamline, before any EXAFS spectrum is recorded, the crystal bender and the goniometer has to be set so as to cover the energy range of the spectra. Then a procedure for energy calibration for that particular setting has to be followed. The usual procedure is to record absorption spectra of two standard samples. The aim of the present work is to study the various aspects of the calibration and to describe a procedure which should be recommended to be followed at this beamline for EXAFS measurements.

After calibration of the particular setting of the polychromator, the performance of the beamline set-up should be checked by recording EXAFS spectra of a standard and then analysing it by fitting with theoretical model. In the present paper, the procedure for such a fitting has also been outlined by taking the example of $\mathrm{K}$-absorption spectra of copper metal foil recorded on this beamline. Extraction of information about the various parameters that can be determined from EXAFS data by the fitting procedure has also been described.

\section{Description of EXAFS beamline at INDUS-2}

The recently developed BL-8 Dispersive EXAFS beamline at $2 \mathrm{GeV}$ Indus- 2 synchrotron source at RRCAT, Indore, India has already been described elsewhere (Das et al 2004, Bhattacharyya et al 2009, Bhattacharyya et al 2009). A schematic diagram describing the basic principles of 


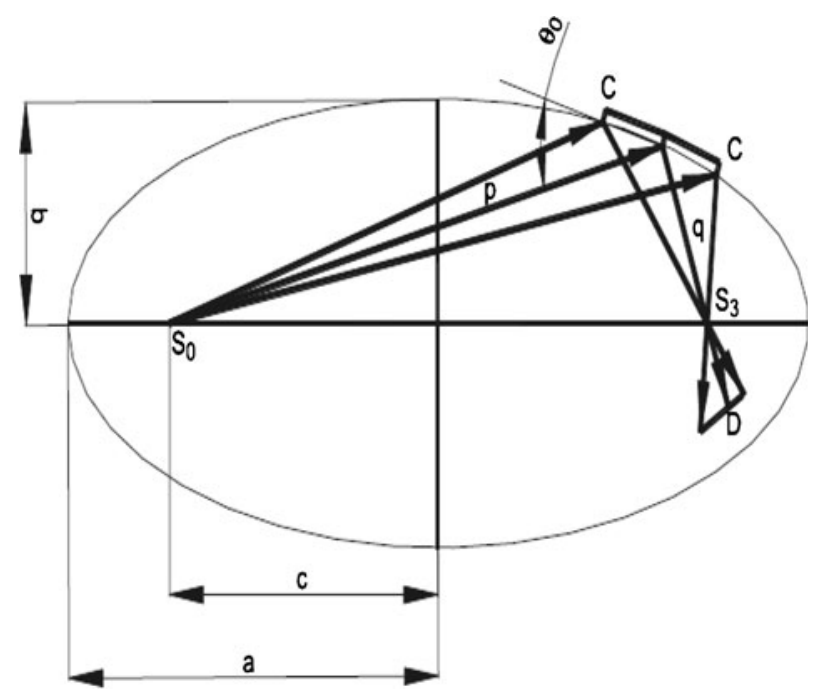

Figure 1. Schematic diagram showing the principle of action of the beam-line.

the beamline is, however, reproduced in figure 1 . The beamline has a $460 \mathrm{~mm}$ long Si (111) crystal (C,C) having $2 \mathrm{~d}$ value equal to $6.2709 \AA$ mounted on an elliptical bender, which can bend the crystal to take shape of an ellipse (Lee et al 1994). The source $\left(\mathrm{S}_{0}\right)$ and the sample positions $\left(\mathrm{S}_{3}\right)$ are situated at two foci of the ellipse. The white synchrotron radiation emerging from one focus $\left(\mathrm{S}_{0}\right)$ is dispersed and focused at the other focus, $\left(\mathrm{S}_{3}\right)$ after reflection from the crystal. The elliptical optics offers minimum aberration. The radiation transmitted through the sample is detected by a position sensitive CCD detector (D) having $2048 \times 2048$ pixels. The whole absorption spectrum can be recorded simultaneously in a short duration of even a few micro seconds in case of a rich sample. The beamline has been designed to cover the photon energy range of 5 to $20 \mathrm{keV}$ providing energy band widths of $0.3 \mathrm{keV}, 1.0 \mathrm{keV}$ and $2.0 \mathrm{keV}$ and with resolutions of $\sim 0.5 \mathrm{eV}, 1 \mathrm{eV}$ and $2 \mathrm{eV}$ per pixel at photon energies of $5 \mathrm{keV}, 10 \mathrm{keV}$ and $20 \mathrm{keV}$, respectively. The plot of absorption versus photon energy is obtained by recording the intensities $\mathrm{I}_{0}$ and $\mathrm{I}_{\mathrm{t}}$, as the CCD outputs, without and with the sample respectively and using the relation, $\mathrm{I}_{\mathrm{t}}=\mathrm{I}_{0} \mathrm{e}^{-\mu \mathrm{t}}$, where $\mu$ is the absorption coefficient and $\mathrm{t}$ is the thickness of the absorber.

\section{Energy calibration using two absorption edges}

The energy calibration of the beamline for a particular setting of the polychromator can be done by recording the absorption spectra of two standards, whose absorption edge energies are wellestablished. First edge energy should be in the beginning and the second edge energy should be at the end of the range of the spectra to be recorded. For example, for recording the absorption spectra of copper samples, the two standards can be $\mathrm{Cu}$ metal foil and $\mathrm{Lu}$ oxide powder. The absorption spectra of $\mathrm{Cu}$ K-edge and $\mathrm{Lu}_{2} \mathrm{O}_{3} \mathrm{~L}_{3}$-edge should be recorded under the same setting of crystal bender and the goniometer. In the present work, we have recorded ten sets of such spectra at different beam currents. One set of spectra recorded at $62 \mathrm{~mA}$ beam current is shown in figures 2(a-c). The CCD channel numbers at which the $\mathrm{Cu}$ metal $\mathrm{K}$-edge and $\mathrm{Lu} \mathrm{L}_{3}$-edge in $\mathrm{Lu}_{2} \mathrm{O}_{3}$ have been found to appear, at different beam currents, have been listed in table 1 . Taking 
(a)

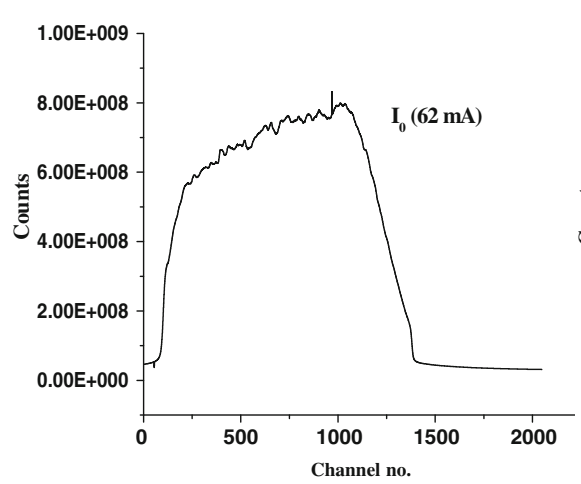

(b)

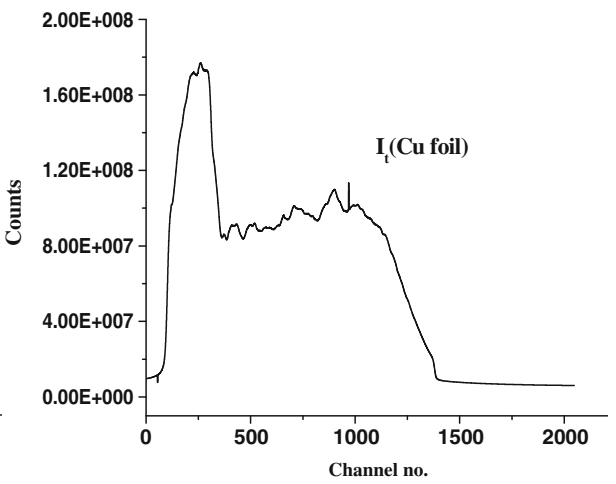

(c)

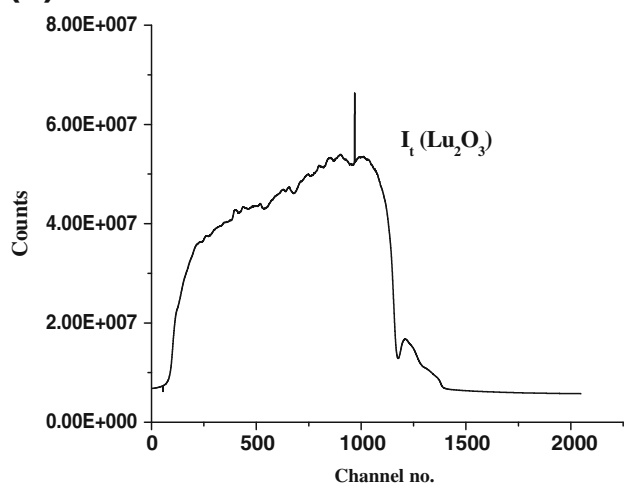

Figure 2. (a) $\mathrm{I}_{0}$ versus channel number, (b) $\mathrm{I}_{\mathrm{t}}$ versus channel number with $\mathrm{Cu}$ metal foil as absorber, (c) $\mathrm{I}_{\mathrm{t}}$ versus channel number with $\mathrm{Lu}_{2} \mathrm{O}_{3}$ as absorber. All the readings are for $62 \mathrm{~mA}$ beam current.

the values of the energies of $\mathrm{Cu} \mathrm{K}$-edge and $\mathrm{Lu} \mathrm{L}_{3}$-edge in $\mathrm{Lu}_{2} \mathrm{O}_{3}$ as $8980.5 \mathrm{eV}$ and $9249 \mathrm{eV}$ respectively (Bearden 1964, Deslattes et al 2003), the CCD channels have been calibrated as follows.

We have used two methods for calibration of the data. In the first method, the position of first maximum of the derivative of absorption curve is taken as the position of the edge energy as shown in figures $3 \mathrm{a}$ and $3 \mathrm{~b}$. In the second method, the position of the point at half edge step in the absorption curve is taken as the position of the edge energy as shown in figures $3 \mathrm{c}$ and d. The required positions of the energies can be found with the help of the software Athena for both of these methods. The values of CCD channel numbers corresponding to the position of edge energy for both $\mathrm{Cu}$ metal foil and $\mathrm{Lu}_{2} \mathrm{O}_{3}$ are measured at different beam currents to observe if there is a variation. After obtaining the channel numbers, corresponding to the position of edge energies of $\mathrm{Cu}$ metal $\mathrm{K}$-edge and $\mathrm{Lu} \mathrm{L}_{3}$-edge in $\mathrm{Lu}_{2} \mathrm{O}_{3}$, their difference is found. Then, the dispersion is obtained by dividing the difference of energies of these edges $(268.5 \mathrm{eV})$ by the difference in channel numbers. The values of the dispersion obtained by the two different methods, at different beam currents, are given in table 1 . The dispersion is required for converting the channel numbers into their corresponding energy values and then obtaining the calibrated curve between $\mu(\mathrm{E})$ and $\mathrm{E}$. 


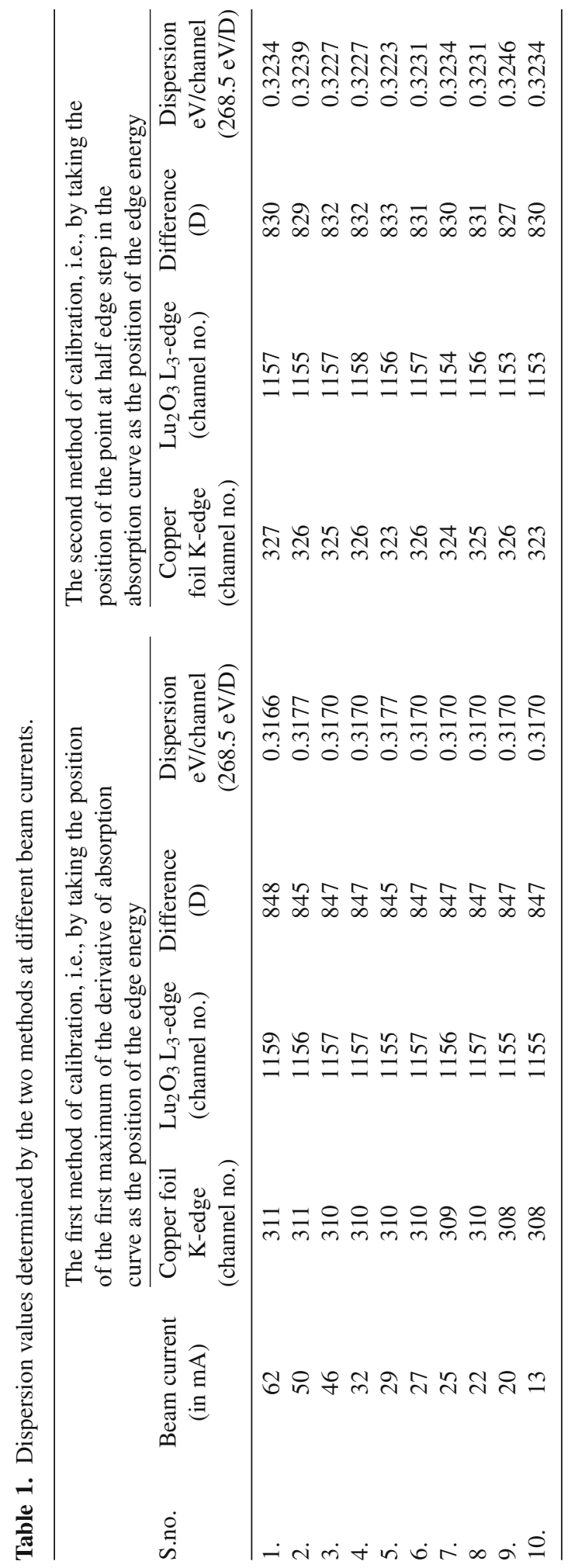


(a)
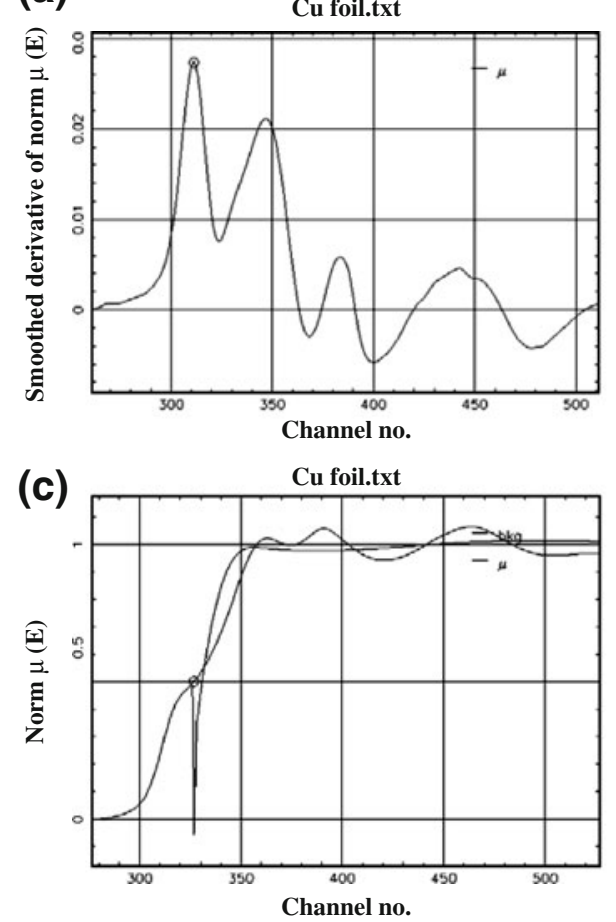

(b)
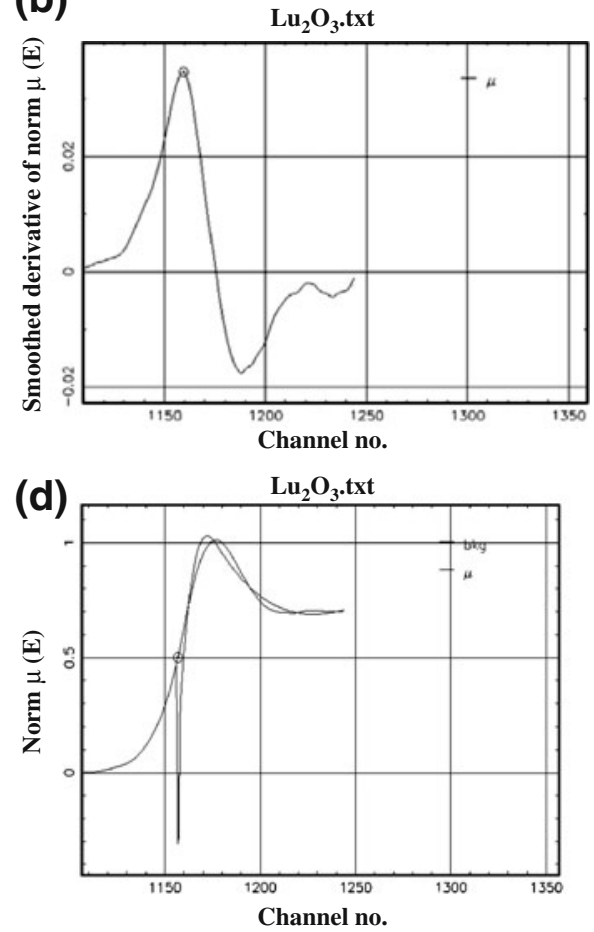

Figure 3. The first method of calibration, i.e., the position of the first maximumof the derivative of absorption curve is taken as the position of the edge energy, is shown in (a) for Cu metal foil and (b) for $\mathrm{Lu}_{2} \mathrm{O}_{3}$. The second method, i.e., the position of the point at half edge step in the absorption curve is taken as the position of the edge energy, is shown in (c) for $\mathrm{Cu}$ metal foil and in (d) for $\mathrm{Lu}_{2} \mathrm{O}_{3}$. The absorption curves were obtained from figure 1.

\section{EXAFS data analysis of copper foil}

To check the performance of the beamline for the particular setting and to outline the procedure for fitting the theoretical model to the calibrated EXAFS spectrum, the copper K-edge EXAFS recorded on the beamline, shown in figure 4 , has been analysed using the available computer software packages Athena version 0.8.061 and Artemis version 0.8.013 (Ravel \& Newville 2005, Kelly et al 2008) (available from website www.xafs.org). These programs include AUTOBK (Newille et al 1993) for background removal, FEFF6L (Zabinsky et al 1995) for generation of the theoretical EXAFS models, and FEFFIT (Newille et al 1995) for parameter optimization of the model. The analysis procedure is as follows.

The spectrum is first normalized by regressing a linear function to the pre-edge region $(-200$ to $-30 \mathrm{eV}$ before the edge energy) and by regressing a linear or quadratic function to the postedge region (50 to $1000 \mathrm{eV}$ above the edge energy). Normalized $\mu(\mathrm{E})$ spectra are produced by subtracting the pre-edge line from the entire data spectrum and then dividing the spectrum by the step height. Normalized $\mu(\mathrm{E})$ data is converted to the $\chi(\mathrm{k})$ data, which is then Fourier transformed.

The Fourier transformed data is then fitted with theoretical model in R-space to obtain the different fitting parameters. These parameters include the passive electron reduction factor $\left(\mathrm{S}_{0}^{2}\right)$, 


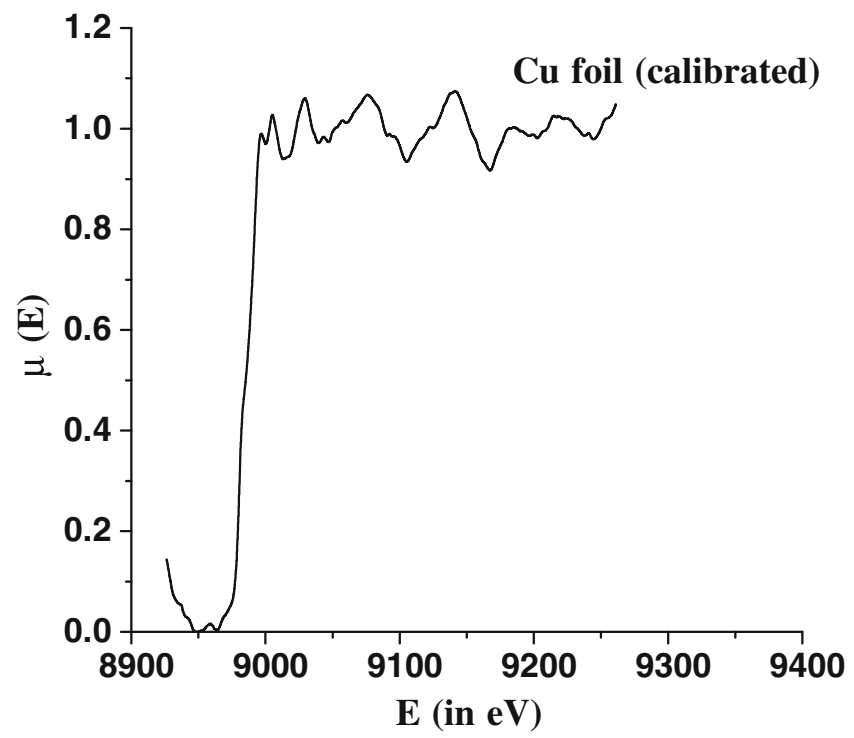

Figure 4. Copper metal foil EXAFS spectrum taken on the beamline for the same setting, calibrated by the first method and then normalized.

the number of identical paths $(\mathrm{N})$, the relative mean-square displacement of the atoms included in path $\left(\sigma^{2}\right)$, an energy shift for each path $\left(\Delta \mathrm{E}_{0}\right)$, and a change in the path length $(\Delta \mathrm{R})$. A single value of $S_{0}^{2}$ and $\Delta E_{0}$ for all the paths is used in the fitting. $\Delta R$ values are defined by an isotropic expansion-contraction term as $\alpha \mathbf{R}_{e f f}$. $\mathbf{R}_{e f f}$ is a special keyword for the effective path length $\mathrm{R}$ for each path from the FEFF calculation of the model structure. This approach is best used

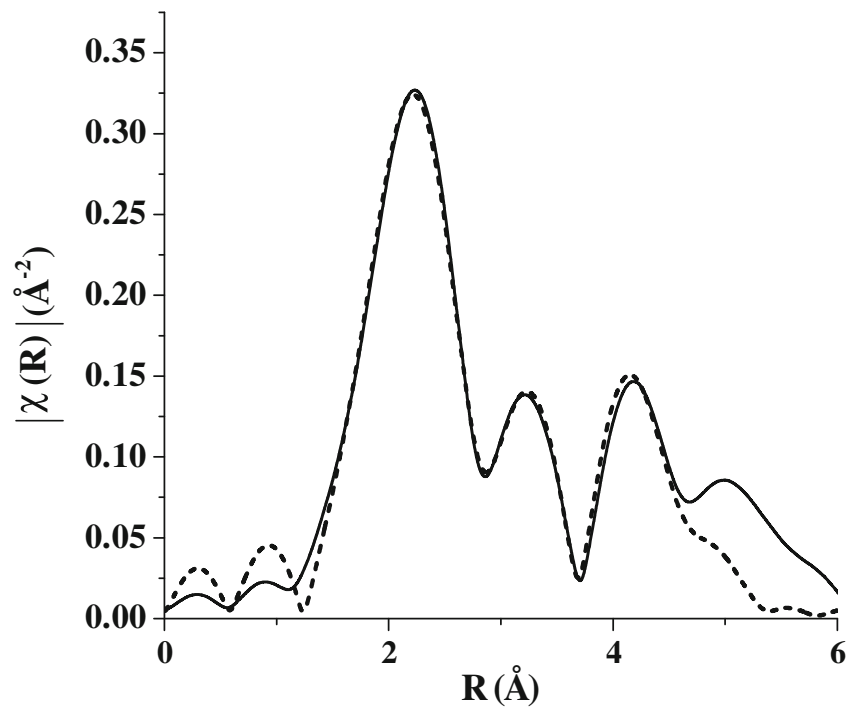

Figure 5. Magnitude of the Fourier transform of the spectrum shown in figure 3 along with the theoretical fit. 
Table 2. The EXAFS results for $\sigma^{2}$ and delr values for $\mathrm{Cu}$ metal foil. For the present work, $\mathrm{S}_{0}^{2}$ was determined to be $0.65 \pm 0.08$. The model constrained the distances $\mathrm{R}$ in terms of an expansioncontraction parameter of $-0.017 \pm 0.003$ relative to the XRD values. The degeneracy of each path $(\mathrm{N})$ is based on the crystal structure of $\mathrm{Cu}$. The best fit value for $\Delta \mathrm{E}_{0}=5.14 \pm 0.84 \mathrm{eV}$. The values of Smith et al are given for comparison.

\begin{tabular}{lccccccc}
\hline Shell & \multicolumn{4}{c}{ Present work } & & \multicolumn{3}{c}{ Smith et al } \\
\cline { 2 - 4 } \cline { 6 - 8 } & $\mathrm{R}(\AA)$ & $\operatorname{del}(\AA)$ & $\sigma^{2}\left(\AA^{-2}\right)$ & & $\mathrm{R}$ & $\mathrm{dell}$ & $\sigma^{2}$ \\
\hline $1^{\text {st }}$ & 2.50 & -0.04 & $0.0068 \pm 0.0015$ & & 2.55 & 0.01 & 0.016 \\
$2^{\text {nd }}$ & 3.54 & -0.06 & $0.0007 \pm 0.0019$ & & 3.60 & 0.02 & 0.022 \\
$3^{\text {rd }}$ & 4.34 & -0.07 & $0.0089 \pm 0.0016$ & & 4.45 & 0.03 & 0.019 \\
\hline
\end{tabular}

for cubic crystal structures like copper in which physical expansions/contractions are likely to be isotropic. A unique Debye-Waller factor $\left(\sigma^{2}\right)$ value is given to each single scattering path in the model. As multiple scattering paths also have important contribution, they are also included in modelling the data. For multiple scattering paths, Debye-Waller factor is defined as the sum of Debye-Waller factors of any two single scattering paths. This is done so as to include the multiple scattering paths without including an extra variable. All the parameters are allowed to vary simultaneously in the fitting procedure so that correlation between all the parameters can be determined. The resulting fitted curve is shown in figure 5 and the parameters so obtained are given in table 2 .

\section{Results and discussion}

In the present work, we have taken the measurements at 10 different beam currents $(62,50,46$, $32,29,27,25,22,20$ and $13 \mathrm{~mA}$ ) but same beam energy $2.5 \mathrm{GeV}$. It can be seen that the values of the dispersion as determined by the first method do not vary much with the values of beam current, i.e., it is almost constant. On the other hand, the values of dispersion, as determined by the second method show more variation with the beam current than the first method. As the dispersion affects the calibration which ultimately affects the data analysis and the results obtained, hence it is advised that the first method outlined by us should be used for finding the dispersion and then for calibrating the experimental spectra. The first method involves taking the position of the first maximum of the derivative spectra of the two standards, for determining their edge positions and energies.

For the analysis of the $\mathrm{Cu}$ metal foil EXAFS data recorded on this beamline, the input parameter $\mathrm{R}_{\mathrm{bkg}}$, that determines the maximum frequency of the background, was set to $1.25 \AA$. Fourier transform was performed over k-range: $\mathrm{k}_{\min }=2.72 \AA^{-1}, \mathrm{k}_{\max }=8.1 \AA^{-1}$. Theoretically modelled data was fitted in the $\mathrm{R}$-space to the experimental data using $\mathrm{k}_{\mathrm{w}}=1$. Fitting was performed only for the first three coordination shells, i.e., in the R ranges of 1.0-5.0 $\AA$. In the fitting procedure, we have used the first 15 paths obtained after FEFF calculation. The value of goodness-of-fit parameter, i.e., reduced chi-square $\left(\chi_{v}^{2}\right)$ is 52.145. The results obtained from fitting are given in table 2 , which gives the local structure parameters obtained from the analysis. The $\mathrm{S}_{0}^{2}$ value so obtained is 0.65 with an error of $\pm 0.08 . \Delta \mathrm{E}_{0}$ value is also reasonable, i.e., $5.14 \mathrm{eV}$ with an error of \pm 0.84 . The value of isotropic expansion-contraction term $\alpha$ as determined from the fit is -0.017 with an error of \pm 0.003 . The delr values determined according to this factor $\alpha$ and are listed in the table. $\sigma^{2}$ factors for multiple scattering paths are defined as the 
sum of $\sigma^{2}$ factors of any two single scattering paths, resulting in three independent $\sigma^{2}$ values. These values are also listed in the table. Our results are comparable with those reported by Smith et al (2006), which are also given in the table.

\section{Conclusions}

Before doing any EXAFS measurements on the recently developed energy dispersive EXAFS beamline at the INDUS-2 synchrotron source, calibration of the particular setting of the polychromator has to be done. It has been shown that the method outlined by us should be used for finding the dispersion and then for calibrating the experimental spectra. This method involves taking the position of the first maximum of the derivative spectra of the two standards, for determining their edge positions and energies. Also, the performance of the beamline for the particular setting should be checked by recording the EXAFS of a standard and by fitting the EXAFS data with a theoretical model, the procedure for which has been outlined. By recording the K-absorption spectra of copper metal foil on this beamline, it has been shown by analysis and extracting information about the various parameters that the performance of the beamline is quite satisfactory.

Thanks are due to Madhya Pradesh Council of Science and Technology (MPCST), Bhopal (India) for a research grant.

\section{References}

Ascone I, Meywr-Klaucke W, Murphy L 2003 Experimental aspects of biological X-ray absorption spectroscopy, J. Synchr. Rad. 10: 16-22

Bhattacharyya D, Poswal A K, Jha S N, Sangeeta and Sabharwal S C 2009 X-ray Absorption Spectroscopy of $\mathrm{PbMoO}_{4}$ crystals, Bull. Mater. Sci. 32: 103-107

Bhattacharyya D, Poswal A K, Jha S N, Sangeeta and Sabharwal S C 2009 First Results from a Dispersive EXAFS beamline developed at INDUS-2 Synchrotron Source at RRCAT, Indore, India, Nucl. Instrum. Meth. A 609: 286-293

Bearden J. A. X-ray wavelengths 1964 U S Atomic Energy Commission, Oak Ridge, Tennessee, p.456 and 468

Das N C, Jha S N, Bhattacharyya D, Poswal A K, Sinha A K and Mishra V K 2004 Design, fabrication and testing of elliptical crystal bender for the EXAFS beam-line at INDUS-II synchrotron source, Sadhana 29 Part 5: 545-557

Deslattes R D, Kessler Jr. E G, Indelicato P, Billy L de, Lindroth E and Anton J, 2003 X-ray transition energies: new approach to a comprehensive evaluation, Rev. Mod. Phys. 75: 35-99

Hricovini K, Padova P De, Quaresima C, Perfetti P, Brochier R, Richter C, Ilakovac V, Heckmann O, Lechevallier L, Bencok P, Fevre P Le, Teodorescu C 2003 Atomic structure and magnetic properties of Mn on InAs(1 0 0). Appl. Surf. Sci. 212-213: 17-25

Kelly S D, Hesterberg D and Ravel B 2008 Methods of Soil Analysis, Part 5, Mineralogical Methods, Chapter 14 ( Medison ; Soil Science Society of America)

Lamberti C, Bordiga S, Bonino F, Prestipino C, Berlier G, Capello L, D'Acapito F, Llabre's I, Xamena F X and Zecchina A 2003 Determination of the oxidation and coordination state of copper on different $\mathrm{Cu}-$ based catalysts by XANES spectroscopy in situ or in operando conditions, Phys. Chem. Chem. Phys. 5: 4502-4509

Lee P L, Beno M A, Jennings G, Ramanathan M, Knapp G S, Huang K, Bai J, Montano P A 1994 An energy dispersive X-ray absorption spectroscopy beam-line, X6A, at NSLS. Rev. Sci. Instrum. 65: 1-6 
Lytle F W 1999 The EXAFS family tree: a personal history of the development of extended X-ray absorption fine structure. J. Synchr. Rad. 6: 123-134

Newille M, Livins P, Yacoby Y, Rehr J J and Stern E A 1993 Near-edge X-ray-absorption fine structure of $\mathrm{Pb}$ : A comparison of theory and experiment, Phys. Rev. B. 47 :14126-14131

Newille M, Ravel B, Haskel D and Stern E A 1995 Analysis of multiple-scattering XAFS data using theoretical standards Physica B. 208 and 209, 154

Ravel B and Newville M 2005 Athena, Artemis, Hephaestus: data analysis for X-ray absorption spectroscopy using IFEFFIT, J. Synchroton Rad. 12: 537-541

Smith A D, Pradell T, Roque J, Molera J, Vendrell-Saz M, Dent A J and Pantos E 2006 Color variations in 13th century hispanic lustre - an EXAFS study, J. Non-Cryst. Solids 352, 5353-536

Smolentsev G, Guilera G, Tromp M, Pascarelli S and Soldatov A V 2009 Local structure of reaction intermediates probed by time-resolved X-ray absorption near edge structure spectroscopy, J. Chem. Phys. 130: 174508

Stern E A 2001 Musings about the development of XAFS. J. Synchr. Rad. 8: 49-54

Tournus F, Masenelli B, Melinon P, Blas'e X, Perez A, Pellkarin M, Broyer M, Flank A M, Lagarde P 2002 Bridging C60 by silicon: Towards non-Van der Waals C60-based materials. Phys. Rev. B 65: 165417165422

Zabinsky S I, Rehr J J, Ankudinov A, Albers R C and Eller M J 1995 Multiple- scattering calculations of X-ray-absorption spectra, Phys. Rev. B. 52: 2995 